\title{
Whole Body MRI Evaluation in Patients with Multiple Myeloma: A Must
}

\author{
Mihaela Buzoianu ${ }^{1}$, Ioana Gabriela Lupescu ${ }^{1,2}$, Emi Marinela Preda ${ }^{1}$
}

Corresponding author:

Mihaela Buzoianu, MD

Department of Radiology and Medical

Imaging, Fundeni Clinical Institute,

Bucharest, Romania

E-mail: mihaela.buzoianu@yahoo.com

Received: 3.07 .2017

Accepted: 15.08 .2018

Copyright $\odot$ Celsius Publishing House www.sgo-iasgo.com
'Department of Radiology and Medical Imaging, Fundeni Clinical Institute, Bucharest, Romania

2"Carol Davila" University of Medicine and Pharmacy, Bucharest, Romania

\section{ABSTRACT}

Magnetic resonance imaging (MRI) is increasingly used in the diagnosis of multiple myeloma (MM). The new statement of the International Myeloma Working Group (IMWG) introduced the role of Whole-body Magnetic Resonance Imaging (WB-MRI) in the management of patients with this haematological disease. We report the case of a hospitalized patient in the haematology department, who was diagnosed with MM and investigated by WB-MRI in order to achieve a complete lesions' report, and staging of the disease. The presented case illustrates more lesions of the spine, long bones and the skull. Common MRI characteristics of MM lesions were defined as a nodular injuries, which were hypointense with T1-weighting, hyperintense with STIR and/or hyperintense with b-800 diffusion-weighting. WB-MRI examination was shown to be a sensitive imaging technique in evaluation of bone marrow and it was included in the DurieSalmon staging system PLUS.

Key words: multiple myeloma, Whole-Body Magnetic Resonance Imaging, MRI findings

\section{PURPOSE}

We aimed to make a review of the literature and illustrate the MRI protocol and the typical findings associated with the correct staging of patients with multiple myeloma.

\section{INTRODUCTION}

Multiple myeloma (MM) is a haematological malignancy characterized by proliferation of monoclonal plasma cells in the bone marrow (1). The disease evolves from an asymptomatic stage, monoclonal gammopathy of undetermined significance (MGUS), and progresses over smouldering myeloma (sMM) to symptomatic MM disease (2).

Imaging evaluation of patients with $\mathrm{MM}$ is very important for the assessment of the extent and severity of disease at presentation, identification of complications and assessment of therapy response (2). Magnetic resonance imaging (MRI) is increasingly used in the diagnosis of multiple myeloma, and the recent consensus statement of the International Myeloma Working Group (IMWG) 
introduced the role of Whole-body Magnetic resonance imaging (WB-MRI) in the management of patients with this haematological disease (3). WB-MRI is recommended in the initial staging of patients with MM due to its high sensitivity in detecting focal lesions or diffuse infiltration of bone marrow, soft tissue injuries, extraosseous lesions as well as for the assessment of treatment response (4). Because it has been clearly demonstrated that WB-MRI examination is the most sensitive imaging technique for evaluating bone marrow, IMWG, Durie and his collaborators have introduced this imaging method in the Durie-Salmon staging system PLUS (3) (table 1).

Recommended protocol of WB-MRI consists of: sequences T1-weighted without FS, STIR, DWI (b-800 diffusion-weighting), T1-weighted with fat suppression (FS) sequences before and after contrast material iv injection (in patients without contraindications for administering gadolinium-based contrast) (4). T2weighted sequences in the sagittal or coronal plane can be optionally used for better highlighting of the vertebral lesions with spinal cord or nerve root compression.
Table 1 - Durie-Salmon Staging System PLUS (5)

\begin{tabular}{ll}
\hline STAGE & WB-MRI \\
\hline MGUS & All negative \\
\hline IA & Normal skeletal survey or single lesion (smouldering) \\
\hline IB & $<5$ focal lesions or mild diffuse disease \\
\hline IIA, IIB & $5-20$ focal lesions or moderate diffuse disease \\
\hline IIIA, IIIB & $>20$ focal lesions or severe diffuse disease \\
\hline
\end{tabular}

\section{CASE REPORT}

A 53 year-old male patient diagnosed with $\mathrm{MM}$ was admitted in the Haematology Department, for complete evaluation and treatment. A WB-MRI examination was performed, evaluation in which were noted multiple anatomical locations of the disease, diffusely spread in the thoracic and lumbar vertebrae, long bones (left femoral epiphysis, theright tibia). These were defined as nodular lesions which appeared hypointense in T1-weighted sequence (fig. 1).

The STIR sequence from the same WB-MRI examination also found the myeloma lesions that were visible on

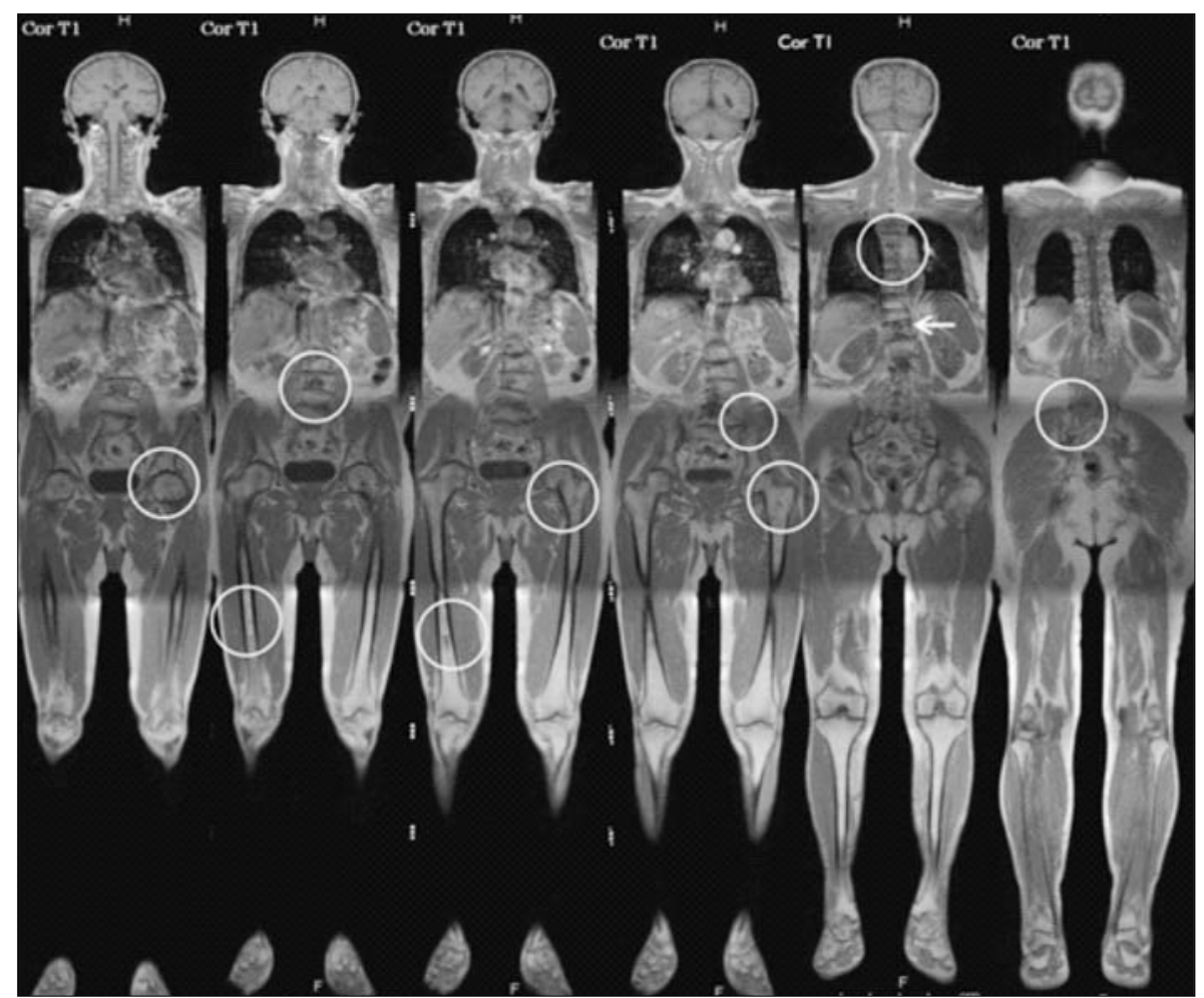

Figure 1 - Multiple myeloma bone lesions: T1 weighted images from WB-MRI exam showing diffusely spread in the thoracic and lumbar vertebrae, long bones, left femoral epiphysis and right tibia, which appear hypointense with T1-weighting. 


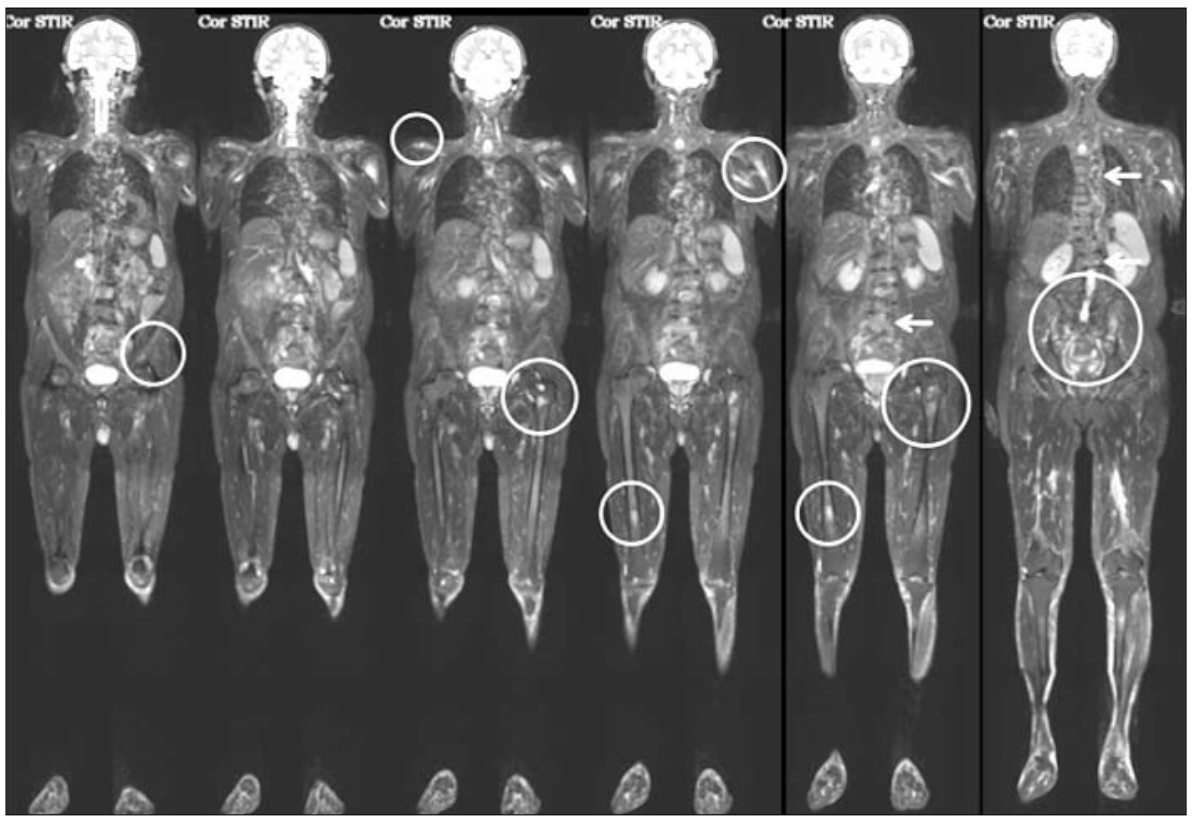

Figure 2 - STIR sequence highlights MM lesions as hyperintense nodules, located on the left humerus metaphyseal-diaphyseal region, on clavicles, right femoral diaphysis, left femoral metaphyseal-diaphyseal region, iliac crests and on the skull.

the T1-weighted sequence. However, in addition to those, lesions smaller than $1 \mathrm{~mm}$ were also traced, which would have been harder to individualise on T1-weighted images. The latter smaller lesions appeared as structured nodular and pseudonodular areas in high signal, located in the left humerus metaphyseal-diaphyseal region, on the clavicles, right femoral diaphysis, left femoral metaphyseal-diaphyseal region, iliac crests and on the skull (fig. 2).

MM injuries previously detected on the T1-weighted and STIR images were certified on the diffusion weighted images (DWI) of the WB-MRI examination, in which the water restriction of the lesions noticed on the left humerus, clavicles and on the ribs arches was observed. Additionally, the axial plane provides a good assessment of the sternum involvement and of other

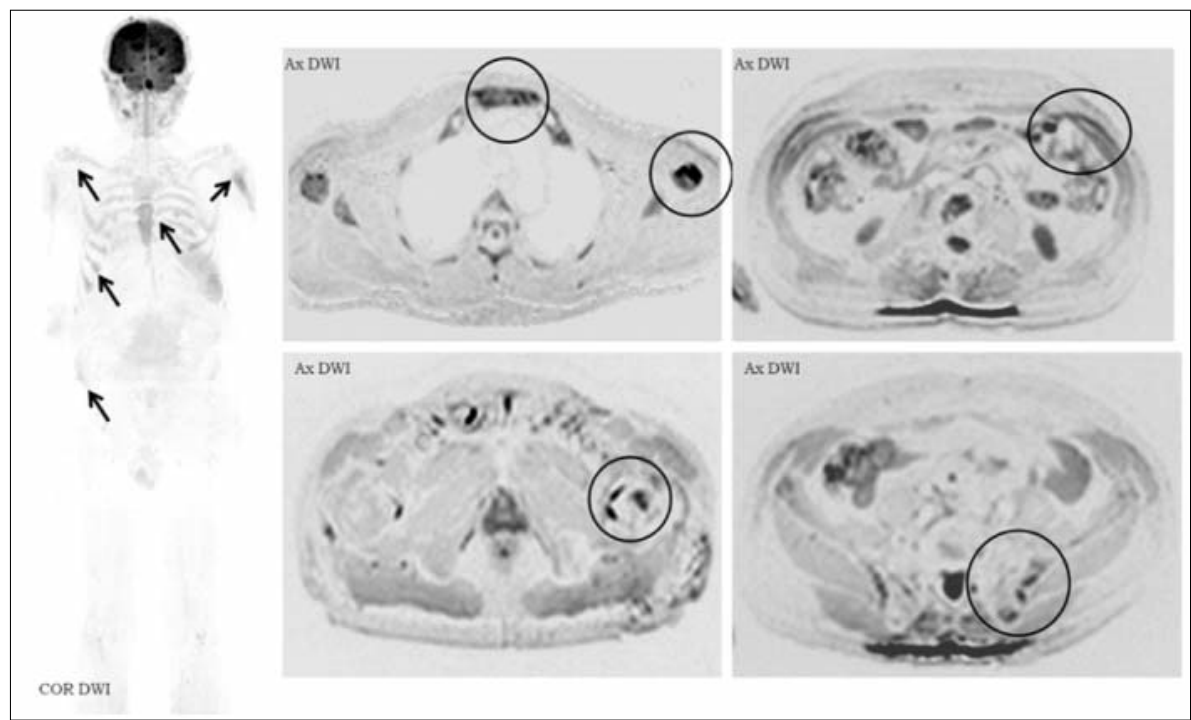

Figure 3 - DWI certifies MM injuries previously described on the T1-weighted and STIR images, revealing the water restriction of the lesions involving the left humerus, clavicles, of the ribs arches, and sternum. 
small lesions identified in the costal arches (fig. 3).

The enhanced T1-weighted sequence with Fat Saturation (FS) showed the enhancement of MM bone lesions, rendering it useful in the assessment of therapy response.

\section{DISCUSSIONS}

According to the literature, the MRI protocols applied to this particular pathology vary between different medical institutions, and may include standard non-enhanced T1- and T2-weighted imaging, STIR sequences and contrast-enhanced T1-weighted fatsaturated imaging (4). In our case we performed an WBMRI protocol that consists in T1-weighted without FS, STIR and DWI (b-800 diffusion-weighting) which offers improved detection of lesions in the spine, pelvis, sternum, skull, humerus, clavicles, ribs arches, femur, and scapulae.

While typical myeloma injuries appear hypointense on T1-weighted images (due to the lower fat content), they are however hyperintense on both fat-suppressed T2-weighted images and on STIR sequences (due to the high cellularity and high amount of water). Typical myeloma injuries develop with predilection in the axial skeleton, spine and pelvis, but they also occur in the extra-axial skeleton, ribs, shoulder, skull and proximal femora (6). This explains the importance of performing a WB-MRI examination in order to assess the extension and staging of the disease.

Different patterns of bone marrow infiltration in the context of MM have been described in the literature, including i. a normal appearing marrow (high signal intensity on T1-weighted and low signal on T2-weighted sequences with fat suppression), ii. focal infiltration (areas of high signal intensity on T2 FS-weighted sequences and low signal intensity on unenhanced T1-weighted images), iii. diffuse disease (a homogenous decrease of signal on T1-weighted images and increased signal intensity on T2-weighted images with fat suppression), iv. salt-and-pepper involvement ( $\mathrm{T} 1$ and T2 weighted images with inhomogeneous "saltand-pepper" pattern) or v. a combined focal and diffuse infiltration (on T1-weighted images, the bone marrow signal intensity is diffusely decreased with additional interspersed foci, which are often better demarcated on fat-saturated images) (6). Four stages are also defined for the diffuse bone marrow infiltration: normal, slight, moderate or severe (table 2).

Regarding the MRI findings in our patient, focal myeloma lesions were noted. These appeared like areas of high signal intensity on T2-weighted sequences and
Table 2 - Stages of diffuse bone marrow infiltration (1)

\begin{tabular}{lccc}
\hline & \multicolumn{3}{c}{ Signal } \\
\hline $\begin{array}{l}\text { Stages of diffuse bone marrow } \\
\text { infiltration }\end{array}$ & T1 & STIR & DWI \\
\hdashline Normal & $\mathrm{N}$ & $\mathrm{N}$ & $\mathrm{N}$ \\
\hline Slight & $\mathrm{N}$ & $\mathrm{N}$ & $\mathrm{H}$ \\
\hdashline Moderate & $\mathrm{N}$ & $\mathrm{H}$ & $\mathrm{H}$ \\
\hdashline Severe & $\mathrm{H}$ & $\mathrm{H}$ & $\mathrm{H}$ \\
\hline
\end{tabular}

Table 3 - Evaluation of overall evolution in MRI (adapted from RECIST criteria) (1).

\begin{tabular}{|c|c|}
\hline Complete response & $\begin{array}{l}\text { Disappearance of the diffuse bone marrow infiltration } \\
\text { (normal signal) and disappearance of the focal } \\
\text { lesions including the most voluminous (1). }\end{array}$ \\
\hline Partial response & $\begin{array}{l}\text { Improvement in the diffuse infiltration and/or reduction } \\
\text { in the number of focal lesions and/or reduction in the } \\
\text { size of the most voluminous focal lesion (1). }\end{array}$ \\
\hline Stable disease & $\begin{array}{l}\text { No criteria either for a response or for disease } \\
\text { progression (1). }\end{array}$ \\
\hline Disease progression & $\begin{array}{l}\text { Aggravation of the diffuse bone marrow infiltration } \\
\text { and/or increase in the number of focal lesions and/or } \\
\text { increase in the size of the most voluminous focal } \\
\text { lesion (1). }\end{array}$ \\
\hline
\end{tabular}

STIR images, low signal intensity on T1-weighted images, with water restriction on DWI. They were predominantly located in the axial skeleton (spine and pelvis), but also in the extra-axial skeleton (ribs, shoulder, skull and proximal femora). The clinical and laboratory findings in correlation with the MRI examination help in staging the myeloma disease. Our patient was staged as having stage III A MM (grade A representing a normal kidney function and normal creatinine values).

The lesions visible on MRI are useful for monitoring the evolution of MM under treatment. According to the literature, WB-MRI assessment of the evolution of MM lesions after therapy is adapted to the RECIST criteria (table 3).

The structured report of a WB-MRI examination for diagnosing and staging of the disease should contain the following criteria: whether myeloma lesions are visible or not; the MRI features of myeloma injuries; the number of the focal injuries or the stage of diffuse bone marrow infiltration; and the location of lesions (axial or extra-axial skeleton); the presence of complications or others incidental lesions. The imagery follow up of multiple myeloma is based on WB-MRI and relies on the following criteria: the number of lesions (whether it increases or decrease in one bone segment or in the whole skeleton), the size of the known lesions, and also the occurrence or not of new lesion(s) or of a complication. 


\section{CONCLUSIONS}

WB-MRI should be considered in all patients diagnosed with $\mathrm{MM}$ and apparently normal conventional radiographs. It should also be considered if there is a suspicion of axial skeletal or extraskeletal lesions, solitary plasmacytoma, as well as in cases in which there is a suspicion of nerve root or spinal cord compression. WB-MRI examination should mandatorily include T1weighted sequences, STIR and DWI images. The STIR sequence is the most sensitive in detecting bone and soft tissue lesions, while T1 weighted sequences are extremely useful in detecting diffuse neoplastic infiltration and enhanced T1 weighted images as well as DWIMRI can be used to evaluate and monitor response after treatment.

\section{REFERENCES}

1. Duvauferrier $R$, Valence $M$, Patrat-Delon $S$, Brillet $E$, Niederberger $E$, Marchand A, et al. Current role of CT and whole body MRI in multiple myeloma. Diagnostic and Interventional Imaging. 2013;94:169-83.

2. Dutoit JC1, Vanderkerken MA, Verstraete KL. Value of whole body MRI and dynamic contrast enhanced MRI in the diagnosis, follow-up and evaluation of disease activity and extent inmultiple myeloma. Eur $\mathrm{J}$ Radiol. 2013;82(9):1444-52. doi: 10.1016/j.ejrad.2013.04.012. Epub 2013 May 28.

3. Baur-Melnyk A, Buhmann S, Becker C, Schoenberg SO, Lang N, Bartl $\mathrm{R}$, et al. Whole-Body MRI Versus Whole-Body MDCT for Staging of Multiple Myeloma. AJR. 2008;190:1097-1104.

4. Derlin T, Bannas P, et al. Imaging of myeloma multiple: Current concepts. World Journal of Orthopedics. 2014;5(3):272-82.

5. Durie BG. The role of anatomic and functional staging in myeloma: description of Durie/Salmon plus staging system. Eur J Cancer. 2006; 42(11):1539-43. Epub 2006 Jun 13.

6. Dutoit JC, Verstraete $\mathrm{KL}$. MRI in multiple myeloma: a pictorial review of diagnostic and post-treatment findings. Insights Imaging. 2016; 7(4):553-69. doi: 10.1007/s13244-016-0492-7. Epub 2016 May 10. 